\title{
Dynamical aspects of the EEG in different psychopathological states in an interview situation: a pilot study
}

\author{
Brigitte Rockstroh *, Hans Watzl, Zbigniew J. Kowalik, Rudolf Cohen, Annette Sterr, \\ Matthias Müller, Thomas Elbert \\ Department of Psychology, University of Konstanz, P.O. Box 5560-D23, D-78434 Konstanz, Germany
}

Received 5 February 1997; accepted 7 July 1997

\begin{abstract}
Dynamical brain states can be characterized by non-linear measures of EEG. The present study shows that critical transitions, i.e., abrupt changes from one dynamic pattern of neural mass activity to another one, may be detected by abrupt variations in local chaoticity. Using an ambulatory device, EEG was recorded from 10 patients with a schizophrenic and two patients with an affective disorder during a series of 25-min interviews. Dynamical aspects, in particular, phase transitions in the EEG-dynamics of the EEG were characterized by means of a measure that continuously estimates the chaoticity of the EEG signal and is thus related to its predictability. Results indicate simpler dynamics of the EEG time series in paranoid-hallucinatory patients, while at the same time these patients tended to exhibit more abrupt transitions/unit of time between different dynamical EEG states. Such sudden phase transitions in brain activity were significantly enhanced prior to expressions of thought disorders that were detected by the interviewer and an observer in the conversation, compared with time periods during the interview without such symptoms. 101997 Elsevier Science B.V.
\end{abstract}

Keywords: Schizophrenia; Thought disorders; Nonlinear dynamics; EEG; Chaos

\section{Introduction}

Efforts to discover and specify electrocortical correlates of cognitive, emotional, and behavioral abnormalities in schizophrenic patients on the basis of spontaneous EEG records and evoked potentials have produced consistent results such as an increased occurrence of abnormal EEG patterns; less alpha and more slow EEG activity; shifts from a center frequency to a lower frequency

\footnotetext{
* Corresponding author. Tel: +49-7531-882085; Fax: +49-7531-882891.
}

in the alpha band; shifts to a higher center frequency in the beta band; and generally smaller amplitudes but more frequency variability (for summaries see Itil et al., 1972; Gruzelier and FlorHenry, 1979; Zahn, 1986; Shagass et al., 1982; Miyauchi et al., 1990; Sponheim et al., 1994; Winterer and Herrmann, 1995). Using depth electrodes, spike and sharp wave discharges below the cortex were reported as early as in the 1940s and 1950s (cf. Small, 1982). However, abnormalities found using depth electrodes could be recorded from patients who had normal records when using standard EEG scalp electrodes (Gibbs, 1939). Therefore, the question arises as to whether 
changes in the scalp EEG might be concealed in the complex dynamic pattern of brain waves not easily accessible to visual inspection of the EEG or conventional power spectra analysis. Measures of the dynamic features of EEG have been described to differ between schizophrenic patients and controls and seem to supplement the results of conventional power spectra analyses, as mentioned above. For instance, a higher frontal ( Elbert et al., 1992) and temporoparietal (Koukkou et al., 1992, 1993) dimensional complexity was described in schizophrenic patients, as compared with healthy controls or neurotics. Lutzenberger et al. (1995) suggest that a right hemispheric enhancement of dimensional complexity in schizophrenic patients is evident, particularly during visual perception. Koukkou et al. (1993) related the higher dimensional complexity of distinct brain regions to a 'dissociated functional EEG state' (p. 403), which might be discussed in the framework of a 'loosened organization of thoughts' (p. 397). In healthy subjects, non-linear measures were found to differentiate mental states (e.g., imagery compared with perception, Lutzenberger et al., 1993, 1995; see also Elbert et al., 1994 for a review). They may therefore also be useful in detecting variations between different dynamical brain states, and may eventually add to attempts to link characteristics of the EEG power spectrum to aspects of schizophrenic psychopathology or symptomatology (e.g., Westphal et al., 1990; Shagass, 1977; Strelets et al., 1993; Gruzelier et al., 1993) such as, for example, formal thought disorders.

While global non-linear measures of brain dynamics (such as dimensional complexity) have been useful to differentiate psychopathological and neurological states or groups (for review see Elbert and Rockstroh, 1993), they cannot depict a rapid change in the dynamical state. It seems possible that local measures of the structure of an attractor might provide a better approach to explore rapidly altering types of cognitive processing. Therefore, a nonlinear measure that was derived to overcome such problems of nonstationarity (Kowalik and Elbert, 1994, 1995) was extracted from the EEG. The measure continuously estimates the chaoticity of the signal and is thus related to its predictability.
Particular alterations in this parameter characterize (critical phase) transitions in the EEGdynamics. These measures were chosen particularly to depict local dynamical states, which might be more adequate to disclose dynamics related to ongoing cognitive processes than to static or global measures.

Another question stimulating the present study was whether results obtained in the laboratory setting can be generalized to more natural life situations, where cognitive processes and the flow of thoughts may be significantly different from the well-controlled conditions in the laboratory. In a first approach, we explored whether EEG could be monitored during the everyday situation of a patient communicating with a partner in a conversation, and whether EEG recordings during such an interview situation would provide any information concerning EEG dynamics parallel to eventually atypical processing - such as certain forms of thought disorders like blocking, derailment, incoherence. For control purposes and to reduce patients' fear of the monitoring devices, EEG was measured simultaneously from both patient and interviewer by means of an ambulatory device. Only one pair of electrodes was used in order to minimize inconvenience within the social situation.

Owing to the pioneering character of the work and a small number of subjects, the present results have pilot character.

\section{Methods and materials}

\subsection{Subjects}

Recordings were obtained from 12 (two female) psychiatric inpatients of the local State Hospital. Six patients (two female) fulfilled the diagnosis of a paranoid-hallucinatory schizophrenia (ICD-10: F20.0); four patients met the criteria of a disorganized subtype (F20.1); two patients suffered from depressive disorder (F32.1, in addition F10.20). For each patient, diagnosis was made upon hospital admission by the responsible psychiatrist on the basis of the PSE (Present State Examination; Wing et al., 1982). The mean age of the patients was 31.5 years with a range of 19-46 years. 
Duration of illness (i.e., years since first admission) varied between 0.3 and 11 years around a mean of 4.3 years. The average duration of previous treatment varied between 5 and 144 weeks, around a mean of 55 weeks. With the exception of one, all schizophrenic patients were under neuroleptic medication with a mean of $382.5 \mathrm{mg} \mathrm{CPZ}$-equivalent (range $86-888 \mathrm{mg}$ ). Three patients were receiving additional anticholinergics (Biperiden) and one patient was receiving Carbamazepine (used in addition to neuroleptics). One of the depressive patients was taking an antidepressant drug (Trimipramin, $175 \mathrm{mg})$, the other neuroleptics $(612.5 \mathrm{mg} \mathrm{CPZ}$ equiv.). Medication differed significantly between patients with the disorganized diagnosis $(120 \mathrm{mg}$ CPZ equiv.) and patients with paranoid-hallucinatory diagnosis $(455 \mathrm{mg} \mathrm{CPZ}$ equiv., $F(1,8)=7.0$ $p<0.05)$. The time interval between diagnosis and interview varied between one and 164 days, around a mean of 37.8 days.

The psychiatric status was assessed by a psychiatrist not involved in the interview or study on the day of the interview but independently from the interview by means of the Brief Psychiatric Rating Scale (BPRS, expanded version with behavioral anchors; Lukoff et al., 1986 ${ }^{1}$ ). The mean BPRS score was 43.5. While the diagnostic subgroups did not differ with respect to hallucinations (which were very low in all groups), formal thought disorders (evaluated on the basis of the BPRS subscales 'unusual thought content' and 'conceptual disorganization') were rated as being slightly, but non-significantly more pronounced in paranoid-hallucinatory patients (mean $7.3 \pm 1.4$ ), compared with disorganized (mean $5.7 \pm 1.2, t=4.7$, $p<0.05$ ) and depressives (mean $4.0 \pm 1.0 ; t<1.5$, $p>0.2$ ).

\footnotetext{
${ }^{1}$ The expanded BPRS with behavioral anchors (Lukoff et al., 1986) allows the evaluation of the target symptoms on the basis of 24 constructs: somatic concern, anxiety, depression, guilt, hostility, suspiciousness, unusual thought content, grandiosity, hallucinations, desorientation, conceptual disorganization, excitement, motor retardation, blunted affect, tension, mannerisms, and posturing, uncooperativeness, emotional withdrawal, suicidality, self-neglect, bizarre behavior, elated mood, motor hyperactivity, and distractability; the intensity on each construct is rated on a 7-point scale (not observed-extremely severe). Interrater reliability and prognostic validity are considered to be adequate.
}

\subsection{Procedure and EEG recording}

EEG was recorded from the central lead $(\mathrm{Cz})$ referred to the right mastoid by means of an ambulatory device specifically designed to have a 16-bit resolution ( KÖLNER VITAPORT System). Amplified within the passband from 1.5 to $50 \mathrm{~Hz}$, data were collected at a rate of $200 \mathrm{~Hz}$. The vertical EOG was obtained to control for eye movement artifacts. Electrodes were affixed about $1 \mathrm{~cm}$ above and below the left eye. EEG and EOG data were stored on a memory card within the ambulatory device. For off-line analysis, data were downloaded on an Apple Macintosh computer where they could be screened for artifacts. Segments free of artifacts were analyzed on a IBM-RISC-R6000 workstation. Recordings were started and obtained simultaneously from the patient and the interviewer (psychologist) by means of two identical devices.

Recordings were obtained while the patient and interviewer (the psychologist) sat opposite to each other in the psychologist's office. The familiar person, the familiar surrounding, and the fact that electrodes were attached to both patient and psychologist were meant to reduce tension and to alleviate the patient's possible mistrust of the EEG recording. ${ }^{2}$ Topics of the $25 \mathrm{~min}$ conversation were daily life on the ward (as a warm-up), items the patient wanted to talk about, and current symptoms or problems. The interview was also monitored by a second trained clinician. His presence served (a) to control the patients' device when necessary; and (b) to be attentive to possible thought disorders - as was the interviewer. Whenever the interviewing psychologist or the second observing clinician noticed thought disorders in the patient's answers, they pressed buttons attached to the recording devices which set a marker on a channel parallel to the EEG and EOG channels without being noticed by the patient. Thus, possible thought disorders such as unusual thought contents, sudden changes in topic,

\footnotetext{
${ }^{2}$ In fact, only two patients refused participation because of aversion against the attachment of electrodes. Usually, the rate of refusal to participate in laboratory experiments is considerably higher.
} 
thought stopping, etc. were marked independently by two persons (interviewer and observing psychologist).

The time period of the interview was determined by the storage capacity of the EEG recording device (about $25 \mathrm{~min}$ ). The conversation was also video-taped for eventual post-hoc evaluation. (A retrospective quantitative analysis of the patients' verbal behavior during the interview on the basis of, e.g., changes in mood, amount of spoken words or duration of pauses turned out to be impossible, because the artifact-free intervals extracted for non-linear analysis could not be matched to the videotaped interview in retrospect. An approximate evaluation of the entire 25-min interviews with respect to time occupied by speaking and listening/thinking during the interview did not reveal noticeable differences between patients' conversational features, e.g., in the amount of spoken phrases or length of pauses, since patients who gave short answers, received more questions. Furthermore, intensive emotional expressions or outbreaks did not occur in any interview.)

\subsection{Data reduction and analysis}

The number and time points of markers set by the interviewer and the observer served to evaluate the interrater reliability of symptom detection, as well as the relationship between symptoms and parameters of EEG dynamics.

From the 12 clinical records (one for each patient) and the 12 records obtained from the interviewer, EEG epochs free of drifts, ocular and movement artifacts with a minimum length of $3 \mathrm{~min}$ were selected through an interactive computer program which permitted visual inspection of the traces. The mean duration of the analyzed epochs were nearly the same with $19.7 \pm 1.5 \mathrm{~min}$ for the patients and $18.9 \pm 4.5 \mathrm{~min}$ for the interviewer from a total recording time of $25 \mathrm{~min}$.

\subsection{The chaoticity measure and the detection of critical transitions}

The differentiation of groups in terms of signal analysis can either be based on static properties of the measured signal (conventional Fourier spectra or correlation functions, fractal dimension, entropy, etc.) or on dynamic characteristics such as Lyapunov exponents (LE) or scanned values of a dimension (Elbert et al., 1994) or entropy rates (Kowalik and Elbert, 1995). 'Jumps', i.e. sudden transitions between local states can appear either with or without a change in the fractal dimension of the particular attractor. Thus, it is not possible to detect changes in a chaotic attractor associated with a corresponding state of the brain estimating the global dimensionality or Lyapunov Exponents (Wolf et al., 1985). However, LEs are strongly differentiated inside the structure of a given attractor. In such a case, if one looks at an attractor locally and regards its stability, one will notice a change of local LE. By introducing a scanning technique to estimate the local LE and by monitoring changes of the average $L E$ value within a scanning window of, e.g., 512 data points, each transition between states will be associated with the rapid changes of the local LE, such that it crosses a zero line within a specific parameter (see Kowalik and Elbert, 1995 for a detailed description of this method). In short, the local LE measures locally, i.e., within some particular region of the attractor, an exponential divergence of chaotic trajectories.

'Chaos' is indicated by neighboring trajectories changing their distance with time. With an iteration number $n$, this can be modeled in the following way:

$\operatorname{dist}\left(X_{0}(n)\right)=\operatorname{dist}\left(X_{0}(0)\right) 2^{\lambda \hat{n} n}$

or by eliminating an exponential function by finding the logarithm of both sides:

$\lambda x_{0 n}=\frac{1}{n} \log \frac{\operatorname{dist}\left(X_{0}(n)\right)}{\operatorname{dist}\left(X_{0}(0)\right)}$

where dist defines the momentary distance of trajectories:

$\operatorname{dist}^{2}(u, v)=\sum_{j=0}^{m}(X[u+j . r]-X[v+j . r])^{2}$

Theoretically, the equation is true only for $n \rightarrow \infty$. Practically, the above rules hold for a small $n$, because in a real system with noise, the system can 'forget' aspects of its dynamics. In order to compromise this internal discrepancy, a stability 
measure such as an averaged LE is considered. The averaging must be limited to a specific set of allowed distances $S_{n}$ :

$i x_{0}=\frac{1}{M} \sum_{S_{n}} \frac{1}{n} i x_{0 n}$

where $M$ is the number of elements in the set $\left\{S_{n}\right\}$ containing indices of 'appropriate distances'. The simplest choice of this averaging set is found by taking the least $M$ values of $A x_{0}$ from $N$ available:

$i x_{0}=\frac{1}{M} \sum_{n=N-M} \frac{1}{n} i x_{0 n}$.

This produces an estimate of the local average value of LE. For the present analyses $M$ was set to 65 and an embedding dimension of 15 was used. ' $n$ ' denotes iterations and ' $A$ ' can thus be given in bits/iteration.

Based on the above definition, the measure of chaoticity is defined as the temporal change of the local LE when the data set is scanned over time. For every time segment the dynamics are reconstructed and the corresponding value of the local LE is computed. This results in a new time series of momentary local LEs. In the present study, a moving average with corresponding standard deviation of this measure of chaoticity was estimated for every patient's and for each of the partner's EEG-record. We refer to this average across the interval $\Delta t$ as ' $A$-chaoticity'. For the present analysis we chose $\Delta t$ to correspond to 512 points.

$A=\langle\lambda\rangle_{\Delta t}$.

Furthermore, $\sigma$-chaoticity, the standard deviation of $A$, was calculated in order to detect nearly periodic behavior. For periodic-like processes (quasiperiodic or periodic), the $\sigma$-chaoticity is drastically reduced. Therefore, this measure operates like a filter for periodic and quasiperiodic behavior, with lower values indicating the presence of nearly periodic processes.

Critical changes of the attractor structure are associated with the crossing of the zero line of the $A x_{0}$ value, as well as with a change of the level of the chaoticity $A$ (see Fig. 1 for examples).

In the present study, the number of critical transitions for every time series was estimated as the number of zero-crossings of the $A$ measure. ${ }^{3}$ For the comparisons of transitions between subjects, the number of zero-crossings of $A$ was referred to unit time (transitions/min) in order to compensate for the variability of analyzed epochs between the two partners in the conversation.

\section{Results}

\subsection{Chaoticity measures}

The paranoid-hallucinatory subgroup exhibited reduced $A$-chaoticity $(0.65 \pm 0.05)$ relative to disorganized $(0.76 \pm 0.1)$ and depressive patients $(0.8 \pm 0.01 ; F(2.9)=5.3, p<0.05)$. Post-hoc analysis (Fisher's PLSD) indicated that the paranoidhallucinatory subgroup differed significantly from both the depressive patients and the disorganized schizophrenics $(p<0.05)$. Given that smaller values of $A$-chaoticity indicate higher predictability of the EEG time series, this would mean enhanced predictability of the EEG time series in paranoidhallucinatory patients. $\sigma$-chaoticity, i.e., the standard deviation of $A$, in the EEG of the psychologist during the interview situation varied depending on the patient. This variability measure was more pronounced when the psychologist was engaged in conversations with paranoid-hallucinatory patients than in conversations with disorganized or depressive patients $(F(2,9)=5.9, p<0.05$; posthoc comparisons indicated this effect to be limited to the paranoid-hallucinatory subgroup, $p<0.01$ ).

\subsection{Critical transitions}

Within the patient group, a tendency for more transitions/min in paranoid-hallucinatory patients $(2.6 \pm 0.7)$ compared with disorganized $(2.0 \pm 1.7)$ and depressive patients $(1.5 \pm 0.8)$ fell short of statistical significance $(t=1.9, \quad p=0.1)$. The number of transitions/min was not significantly related to medication $(r=0.11)$ or general thought

\footnotetext{
${ }^{3}$ The rapid change of the dynamics is associated with a divergence in the $A$-pattern. The choice of a particular threshold makes it possible to "count" such a divergence as a transition between dynamical states. For the present analysis, the zero line was chosen as threshold.
} 

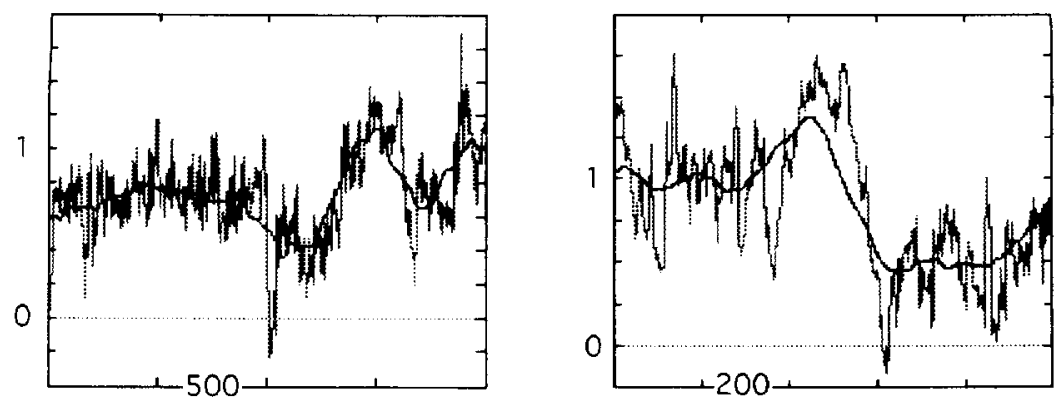

Fig. 1. Examples of changes of dynamics in a EEG recording epoch of $500 / 200 \mathrm{sec}$ each obtained from a schizophrenic patient (paranoid--hallucinatory diagnosis). Note the change of the $A$-chaoticity before and after critical transitions (dark line). Critical transitions are indicated by the zero crossing of the LE course. A zero crossing of the momentary chaoticity, 'A-chaoticity', like a negative LE can be considered an indicator of a change in the system's dynamical behavior.

disorders (BPRS subscales 'unusual thought content' and 'conceptual disorganization': $r=0.54$, $p=0.13$ ).

The relationship of dynamic measures is illustrated in Fig. 2 for the patient subgroups: patients with paranoid-hallucinatory diagnosis exhibit enhanced predictability but also more

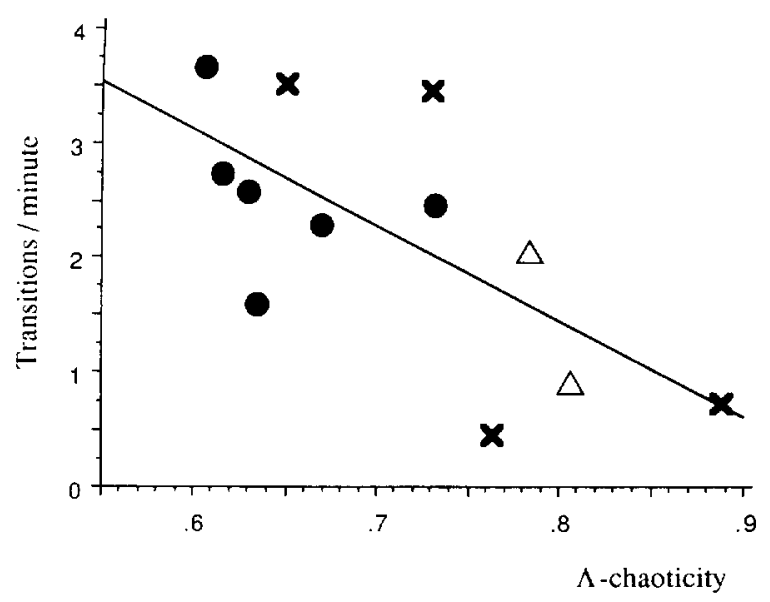
Patients with the diagnosis of
- paranoid-hallucinatory subtype
$\boldsymbol{X}$ disorganized subtype
$\triangle$ major depressive disorder

Fig. 2. Relationship between chaoticity (abscissa) and number of transitions/min (ordinate) in patient subgroups. Patients with a paranoid-hallucinatory diagnosis (filled circles) exhibit lower EEG-A-chaoticity (abscissa) and a tendency of more transitions/min (ordinate) than depressives (triangles). ' $\mathrm{X}$ ' represent the four patients with a disorganized diagnosis. The lower value for A-chaoticity indicates higher predictability. transitions/min than depressive patients, while a variable pattern is evident for schizophrenic patients of the disorganized type.

\subsection{Relationship of EEG dynamics and expression of thought disorders}

Setting of markers, i.e., detection of thought disorder indices by the psychologist engaged in the conversation and the observing psychologist was related between the two $(r=0.67$; Fisher's = $p<0.05$, for the number of markers). Diagnoses did not seem to have affected the detection rates of either one. The rating of thought disorders according to the BPRS subscales ('unusual thought content' and 'conceptual disorganization') was significantly $(r=0.89, p<0.01)$ related only to the number of markers set by the observer.

The relationship between the EEG dynamics, indicated by the number of transitions (zero-crossings of the LE) in the EEG, and symptoms, indicated by markers set by both psychologists, was examined in the following way: only the number of markers set by both raters within $1 \mathrm{~min}$ were considered. This resulted in $0-7$ markers/ patient (with 0 and 1 for the two depressive patients). The mean interval between the markers of the interviewer and observer amounted to $27.5 \pm 15 \mathrm{~s}$ (median $28 \mathrm{~s}$ ). There was an average of $3.9 \pm 2.2$ (median 3 ) transitions within 1 min prior to these markers, a number which is significantly larger $(t(20)=2.2, p<0.05)$ than the average occurrence of transitions during the entire interview. To 
clarify this point further, the number of transitions within $1 \mathrm{~min}$ was also established for 'control' intervals of $1 \mathrm{~min}$ that were chosen so that no marker was set within a minimum of 1 min around this interval. For these control intervals, the number of transitions/min was $1.5 \pm 2.1$ (median 1.5). A comparison of the number of transitions between these 'critical' and 'control' intervals revealed a significant difference with $t(16)=3.4, p<0.01$. The average interval between marker and preceding transition was $25.4 \mathrm{~s}$ (median $23.3 \mathrm{~s}$ ).

\section{Discussion}

The present results seem to be promising in two respects: firstly, they demonstrate the possibility of monitoring the EEG under realistic conditions, thereby enabling a closer examination of electrocortical correlates not only of general symptomatology or characteristic cognitive processes in schizophrenic patients under real life circumstances, but also of characteristics in the interaction of conversation partners. Secondly, dynamical aspects of the EEG may be related to such characteristic cognitive or behavioral processes.

The $A$-chaoticity can be considered a measure of short-term predictability (Kowalik and Elbert, 1994, 1995). while the density of the phase transitions is inversely proportional to the long-term stability of the system. For periodic-like processes (quasiperiodic or periodic), the $\sigma$-chaoticity is drastically reduced. Therefore, this measure operates like a filter for periodic and quasiperiodic behavior, with lower values indicating the presence of nearly periodic processes. The present results suggest that short-term predictability of the EEG time series over seconds, i.e., within the scanning window of 512 points each. was increased in medicated patients of the paranoid-hallucinatory subgroup compared with the other subjects. On the other hand, more instability of the brain dynamics, the EEG time series during the 25-min conversation was indicated by the tendency for an increased rate of critical transitions in the same patient group. If this result can be confirmed, it suggests that (medicated) schizophrenic brain activity is characterized by more rapid changes in temporary dynamical states, while each of these states are 'simpler' in terms of its chaoticity, i.e. its attractor structure, which also means that these states are more predictable than the waking states of controls. For controls, simpler attractors are generally observed during the relaxed waking state or during sleep stages (e.g. Elbert et al., 1994). while so far, little is known about the non-stationarity, or the rate of critical transitions, respectively. The present data set is too limited to allow conclusions, as to what extent this interplay of brain dynamics could have contributed to the characteristic symptom pattern suggested by the diagnosis and the BPRS ratings. However, the indication that a diagnostic subgroup can also be described by a pattern of dynamical measures should be extended and further explored.

Sudden changes in brain dynamics, as depicted by 'jumps' in the LE, were also present in the EEG of the interviewer. Currently, we know very little about the relation between transitions in EEG dynamics and behavioral and cognitive variables. Healthy subjects, too, may show transitions--as did the interviewer in the present study $(1.5 \pm 0.2$ on average). It remains to be further experimentally investigated as to what extent sudden phase transitions occur as a result of 'normal' brain processes during a conversation (involving thinking, talking, and listening). It is possible that usually occurring interruptions, such as the "natural' ending of a chain of thoughts, might produce critical transitions which are not categorized as 'symptomatic'. Even the ending of a simple sentence may have caused a transient change in the dynamic flow of the EEG. Therefore, it may be assumed that normally occurring critical transitions exist in EEG for both interviewer and patient. However, schizophrenics, and particularly paranoid-hallucinatory patients, tended to exceed the range of phase transitions established by interviewer and the depressive patients. Furthermore. an increase in transitions might be related to symptoms of formal thought disorders. We must assume a latency variability between a specific change in the brain s dynamics and its expression in some symptom of formal thought disorder. as well as its detection by the listener, and, therefore, only a very global relationship can be expected. Yet for a rather strict selection of events and control intervals, we found symptoms of formal 
thought disorders to be preceded by changes in brain dynamics. Although the present data would be too limited to arrive at the conclusion that transitions are an electrocortical correlate of thought disorders, they should stimulate further exploration in this respect.

An unexpected, yet interesting finding was that the $\sigma$-chaoticity was increased in the interviewer while he was communicating with paranoid-hallucinatory patients, compared with his interviews with the other patients. When communicating with paranoid-hallucinatory patients and paying attention to the occurrence of characteristic symptoms, the brain of the interviewer seems to be in a different dynamical state than during interactions with depressive and disorganized patients. Brain dynamics are sensitive to the characteristics of the interaction. Applying the measure of transitions to examine non-linear dynamics of the client-therapist interaction within and across psychotherapeutics (intensity ratings of categories of strategic purposes and emotional schemata of client and therapist constituting the time series in which transitions were determined in a similar way as described for the present study), Schiepek and Kowalik (Schiepek et al., 1997; Kowalik et al., 1997) observed transitions often occurring in parallel between therapist and client. This result, too, suggests temporal coupling of (brain or behavior) dynamics within interactions.

Although preliminary, the relationship between sudden changes of dynamics and overt events, i.e., thought disorders determined by the interviewing and the observing psychologists, seem of particular interest for two reasons. Firstly, it suggests that distinct cognitive events or processes that manifest themselves as particular in a conversation might have an electrocortical correlate in distinct brain dynamics. This speculation must certainly be validated. However, the scanning of dynamical measures seems to offer more direct access to such a linking of cognitive and brain events within the flow of information processing rather than 'static' averaged EEG power spectra or dimensionality measures. ${ }^{4}$ Secondly, this relationship-at first

\footnotetext{
${ }^{4}$ Critical transitions may be but are not necessarily linked to a change in the power spectrum, and therefore, conventional FFT-scanning with a sliding window would not necessarily detect transitions.
}

glance - did not seem to be bound to a diagnostic category, i.e., it was not only found in patients with a paranoid-hallucinatory diagnosis. The analysis of temporal relations between sudden changes in brain dynamics and cognitive states such as thought disorder, however, should also include the quantitative attributes of verbal interaction, e.g., number of words, pauses in conversation, changes in attention and affect, etc.

In summary, the present pilot results should encourage us to further examine electrocortical correlates of characteristic cognitive processing in schizophrenic patients by recording EEG under such circumstances that these characteristics can be properly revealed, (i.e., during a non-structured and relaxed interview, rather than in a strict laboratory setting) and by evaluating measures which depict alterations in electrocortical dynamics rather than those which characterize stationary states, like average power spectra.

\section{Acknowledgment}

Research was supported by the Deutsche Forschungsgemeinschaft.

\section{References}

Elbert, T., Lutzenberger, W., Rockstroh, B., Berg, P., Cohen, R., 1992. Physical aspects of the EEG in schizophrenics. Biol. Psychiatry 32, 595-606.

Elbert, T., Rockstroh, B., 1993. Das chaotische Gehirn-Zur Erfassung nichtlinearer Dynamik aus physiologischen Zeitreihen. Verhaltensmodifikation und Verhaltensmedizin $14,80-95$.

Elbert, T., Ray, W.J., Kowalik, Z.J., Skinner, J.E.. Graf, K.E., Birbaumer, N., 1994. Chaos and physiology-deterministic chaos in excitable cell assemblies. Physiol. Rev. 74, 1-47.

Gibbs, F.A., 1939. Cortical frequency spectra of schizophrenic, epileptic and normal individuals. Trans. Am. Neurol. Assoc. $65,141-144$.

Gruzelier, J.H., Flor-Henry, P. (Eds.), 1979. Hemisphere Asymmetries of Function in Psychopathology. Elsevier, Amsterdam.

Gruzelier, J.H., Jutai, J.W., Connolly, J.F., Hirsch, S.R., 1993. Cerebral asymmetries and stimulus intensity relationships in EEG spectra of VEPs in unmedicated schizophrenic patients: relationships with active and withdrawn syndromes. Int. J. Psychophysiol. 15, 239-246. 
Itil, T.M., Saletu, B., Davis, S., 1972. EEG findings in chronic schizophrenia based on digital computer period analysis and analog power spectra. Biol. Psychiatry 5, 1-13.

Koukkou, M., Lehmann, D., Wackermann, J., Dvorak, L., Henggeler, B., 1992. The dimensional complexity of the EEG in untreated acute schizophrenics, in persons in remission after a first schizophrenic episode, and in controls. Schizophr. Res. 6, 129

Koukkou, M., Lehmann, D., Wackermann, J., Dvorak, I., Henggeler, B., 1993. Dimensional complexity of EEG brain mechanisms in untreated schizophrenics. Biol. Psychiatry 33, 397-407.

Kowalik, Z.J., Elbert, T., 1994. Changes of chaoticness in spontaneous EEG/MEG. Integrative Physiol. Behavioral Sci. $29,270-282$.

Kowalik, Z.J., Elbert, T., 1995. A practical method for measuring the chaoticity of electric and magnetic brain activity. Bifurcation and Chaos 5, 475-490.

Kowalik, Z.J., Schiepek, G., Kumpf, K., Roberts, L.E., Elbert, T., 1997. Psychotherapy as a chaotic process (Part II): The application of nonlinear analysis methods on quasi time series of the client-therapist interaction: A nonstationary approach. Psychotherapy Research 3 (in press).

Lukoff, D., Liberman, R.P., Nuechterlein, K.H., 1986. Symptom monitoring in the rehabilitation of schizophrenic patients. Schizophr. Bull. 12, $578 \cdot 602$.

Lutzenberger, W., Elbert, T., Ray, W.J., Birbaumer, N., 1993. The scalp distribution of the fractal dimension of the EEG and its variation with mental tasks. Brain Topography 5 , $27-34$.

Lutzenberger. W., Stevens, A., Bartels, M., 1995. Do schizophrenics not differentiate between perception and imagination? Neurosci. Lett. 199, 119-122.

Miyauchi, T., Tanaka, K., Hagimoto, H., Miura, T., Kishimoto, H., Matsushita, M., 1990. Computerized EEG in schizophrenic subjects. Biol. Psychiatry 28, $719-728$.

Schiepek, G., Kowalik, Z.J., Schütz, A., Köhler, M., Richter, K., Strunk, G., Mühlnickel, W., Elbert, T., 1997.
Psychotherapy as a chaotic process. Part I: Coding the Client-therapist interaction by means of sequential plan analysis and the search for chaos: a stationary approach. Psychotherapy Research, 2 (in press).

Shagass, C. 1977. Twisted thoughts, twisted brain waves? In: Shagass, C., Gershon, F., Friedhoff, A. (Eds.), Psychopathology and Brain Dysfunctions. Plenum Press, New York, pp. 353-378.

Shagass, C., Roemer, R.A., Straumanis, J.J., 1982. Relationships between psychiatric diagnosis and some quantitative EEG variables. Arch. Gen. Psychiatry 39, 1423-1435.

Small, J.G., 1982. Psychiatric disorders and EEG. In: Niedermeyer, E., Lopes da Silva, F. (Eds.), Electroencephalography, 2nd edn. Baltimore: Urban and Schwarzenberg, pp. 439-453.

Sponheim, S.R., Clementz, B.A., Iacono, W.G., Beiser, M., 1994. Resting EEG in first-episode and chronic schizophrenia. Psychophysiology 31, 37-43.

Strelets, V.B., Avin, A.J., Arbenkova, O.K., 1993. Topography of brain electric potentials in depressive patients and schizophrenics with disturbances of the thinking process. Hum. Physiol. 19, 46-49.

Westphal, K.P., Grözinger, B., Diekmann, V., 1990. Slow theta activity over the midfrontal cortex in schizophrenic patients. Acta Psychiatr. Scand. 81, 132-138.

Wing, J.K., Cooper, J.E., Sartorius, N., 1982. Die Erfassung und Klassifikation psychiatrischer Symptome. Beltz, Weinheim.

Winterer, G., Herrmann, W.M., 1995. Über das Elektroenzephalogramm in der Psychiatrie: Eine kritische Bewertung. Zeitschrift EEG-EMG 26, 19-37.

Wolf, A., Swift, J.B., Swinney, H.L., Vastano, J.A., 1985. Determining Lyapunov exponents from a time series. Physica 16D, 285-317.

Zahn, T.P. 1986. Psychophysiological approaches to psychopathology. In: Coles, M.G.H., Donchin. E., Porges, S. (Eds.), Handbook of Psychophysiology. Plenum Press, New York, pp. 508-610. 